\title{
Challenges of probe cocktail approach for human drug-drug interaction assays
}

\author{
Daniel B Carrão1, Anderson RM de Oliveira' ${ }^{1}$ \& Igor RS Magalhães ${ }^{*, 2}$ \\ ${ }^{1}$ Laboratório de Metabolismo In Vitro e Técnicas de Separação, Departamento de Química, Faculdade de Filosofia, Ciências e \\ Letras de Ribeirão Preto, Universidade de São Paulo (FFCLRP-USP), 14040-901, Ribeirão Preto, Brazil \\ ${ }^{2}$ Núcleo de Estudos em Farmacocinética, Faculdade de Ciências Farmacêuticas, Universidade Federal do Amazonas (FCF-UFAM), \\ 69077-000, Manaus, Brazil \\ *Author for correspondence: Tel: +55 923305 1181; imagalhaes@ufam.edu.br
}
"Although there are challenges to be overcome, probe cocktail approach is a worthful solution for assessing in vitro DDIs of drug candidates."

First draft submitted: 16 September 2018; Accepted for publication: 24 September 2018; Published online: 7 November 2018

Keywords: CYP450 • drug-drug interaction • HLM • in vitro models • mass spectrometry • N-in-one • probe cocktail

\section{Drug-drug interactions in the early drug development}

In the preliminary stages of drug development, assessing the drug candidate metabolic behavior and its drug-drug interactions (DDIs) potential in humans is mandatory. The US FDA preconizes the evaluation of the drug candidate as substrate, inhibitor and inducer of the human metabolizing enzymes [1]. CYP450 enzymes are responsible for the metabolism of approximately $75 \%$ of market drugs and most of other xenobiotics [2]. Xenobiotics may change CYP450 enzyme action, either by decreasing (inhibition) or by increasing (induction) its activity, which may directly impact the levels of therapeutic agents in the human body and thus compromising effectiveness of the treatment [3-7]. This phenomenon may cause severe outcomes as the loss of desired effect or trigger of adverse effects when drugs are coadministrated $[4,7]$. CYP450 inhibition or induction may represent a greater risk for drugs with narrow therapeutic window, once minor changes in their concentration can enhance toxic effects or result in loss of the drug effect.

Nowadays, DDIs are the major cause of drug withdrawal from clinical studies or even from the market [2]. To avoid this failure, the preliminary knowledge of risk interaction from a drug candidate in the early stages of the drug development is essential $[4,6]$. The FDA recommends DDI assays for the major human hepatic CYP450 isoforms, as follows: 1A2, 2B6, 2C8, 2C9, 2C19, 2D6, 3A4 [1]. However, it is also recommended to assess DDIs of other CYP450 isoforms, which may be involved in drug metabolism (2A6, 2E1, 2J2 and others) [4]. For in vitro DDI assays, human liver microsomes (HLM) model is highly employed and the FDA preconizes selective and specific probe substrates for evaluation of each human CYP450 isoform [1]. HLMs are an useful in vitro model for early estimation and prediction of the DDIs potential of the drug candidate [2]. In in vitro studies, the probe substrate and the drug candidate are incubated with HLMs or other model and the enzymatic activity of each CYP450 isoform is determined by monitoring the formation of the respective metabolite (marker). The FDA recommends that DDI methods must be validated employing selective chemical inhibitors for each CYP450 isoform [1]. Although the in vitro models provide important information of DDIs potential, this approach do not dismiss the need to carry out in vivo assays. In more advanced stages of drug development, DDI in vivo studies are performed by administration of the probe substrate and the drug candidate to subjects, followed by analysis of human plasma or urine samples for quantification of the respective marker [8].

\section{Probe cocktail approach \& its challenges}

Traditionally, DDI studies have been performed by evaluating individually each CYP450 isoform [9]. Although these studies have been providing reliable information, assessing each CYP450 isoform individually has some disadvantages such as the great number of samples, time consuming, labor intense and cost-ineffective [4]. As an alternative, DDI studies employing probe cocktail substrates, also known as 'n-in-one' studies, have been 
reported [10]. The cocktail method is performed by incubating simultaneously several probe substrates. Since one can evaluate all the CYP isoforms or the most of them, this methodology has led to faster and less expensive analysis and thus increasing the efficiency of the DDI studies [3]. However, some challenges employing probe substrate cocktail must be addressed and discussed: "what about the selectivity and specificity of the probe substrate for each CYP450 isoform?" "Are there interactions among the probe substrates?" and "are the analytical methods selective and sensitive enough to analyze all the substrates and metabolites simultaneously?"

The selection of a specific probe substrate for each CYP450 isoforms is important because multiple enzymes may be involved in the metabolism of a xenobiotic [2]. The probe substrate must be selected according to regulatory agencies recommendations, highly specific CYP450 reaction markers and low interaction potential with other probe substrates [3]. The FDA recommends a list of probe substrates which may be used to evaluate each CYP 450 isoform activity through specific metabolism reactions [11]. The specificity and interaction potential of a probe substrate may be related to its concentration. Obtaining a single incubation condition for all probe substrates has been the major issue to implement cocktail probe approaches due to turnover differences among probe substrates $[3,12]$. For low-turnover substrates, higher concentrations are necessary along with high protein concentration and incubation time [3]. This feature indirectly increases the potential of DDIs among the probe substrates and also promotes a disadvantage for high-turnover substrates, respectively [3]. To overcome that, some studies have been reporting the separation of the probe substrates into different cocktail incubations [12]. On the other hand, the development of highly selective and sensitive analytical methods has been overcoming these challenges [7].

It is important to highlight that highly selective and sensitive analytical techniques are fundamental to perform DDI studies employing the probe substrate cocktail approach. Prior to the analysis, it is necessary to preparesamples to make them compatible with the analytical technique and to achieve better limits of quantification (clean-up and preconcentration). Overall, sample preparation techniques most commonly employed in probe cocktail assays are protein precipitation [2-4,6,13], liquid-liquid extraction [14,15], and solid-phase extraction [9].

Among the separation techniques, almost all the studies have employed liquid chromatography analysis [2-9,12]. There are only two articles relating other separation techniques, one employing gas chromatography [14] and one employing supercritical fluid chromatography [13]. Advances in liquid chromatography observed in recent years have also enabled the development of methods capable of achieving the needs of probe cocktail studies, that is, analyzing several analytes in a short period. To accomplish this, the use of columns capable of separating approximately dozens of compounds with very high efficiency is necessary [7]. In the literature, analytical columns with some special properties have been employed for this purpose lately. For instance, columns produced with core-shell technology have been reported [2]. In this case, core-shell particles as stationary phases are employed in columns rather than fully-porous particles, resulting in the improvement of chromatographic behavior [16]. Another example is the use of polar-embedded phases instead of classical straight chain silicas. Spaggiari $e t$ al. took advantage of using a column with an embedded carbamate group in the bonded phase ligand to achieve the separation of 22 analytes [3]. Besides offering an alternative selectivity profile, the polar-embedded stationary phases have been developed to surpass some downsides of conventional alkylsilica phases, such as poor peak shape of basic analytes and low stability in highly aqueous mobile phases [17]. Certainly, these and other improvements in analytical separations will continue to be employed in the development of novel assays devoted to the tentative investigation of drug metabolism.

In the last five years, all reported probe cocktailmethods have employed chromatography techniques coupled to mass spectrometry [2-9,12-14]. Most of the reported methods have employed triple quadrupole mass spectrometers [2$4,9,12]$. There are also manuscripts reporting the use of single quadrupole [13] or high-resolution mass spectrometers [5] to perform probe cocktail assays. Liquid chromatography coupled to mass spectrometry has been and certainly will continue to be the best analytical choice to perform probe cocktail assays. More specifically, ultra-HPLC (UHPLC) coupled to triple quadrupole mass spectrometer is becoming a fundamental analytical technique to overcome the challenges of probe cocktail approach. The high selectivity, sensitivity and resolution of UHPLCMS/MS has allowed to employ lower probe substrate concentrations and low protein concentration and incubation time, avoiding the interactions among the substrates, maintaining their specificity and also minimizing nonspecific microsomal binding [7].

As DDIs probe cocktail assays provide quantitative results, it is also important to highlight that is mandatory to perform analytical method validation before employing the analytical method in probe cocktail studies. Although all the reported works have validated the probe cocktail assays employing selective chemical inhibitors for each CYP450 isoform and also have compared the results obtained with single probe substrate incubations, not all of 
them have performed analytical method validation $[3,13,18]$. The EMA preconizes the analytical method validation assessing its linearity, selectivity, carryover effect, lower limit of quantification, precision and accuracy intra- and interassay, matrix effect and stability [19]. To ensure the reliability of the obtained quantitative results, it is essential to validate the analytical method [20].

Comprehensive DDI studies for drug candidates can be performed employing probe cocktail assays. There are several works reporting DDIs' cocktail methods up to 11 -in-1 incubation [2-10,12-15,18]. Probe cocktail assays can be employed in direct and time-dependent inhibition assays, which are the major causes of CYP450 enzymes inhibition [6]. Direct inhibition may occur through reversible interactions between enzyme and inhibitor and it is important to determine its enzymatic inhibition mechanism and enzymatic inhibition constant $\left(\mathrm{K}_{\mathrm{i}}\right)$ [21]. Time-dependent inhibition are related to irreversible inhibition of CYP450 enzymatic activity and it is known to be the major cause of DDIs [6]. Irreversible inhibitors inactivate the enzyme making necessary the synthesizes of new enzymes to recover the enzymatic activity [21]. The most common irreversible inhibition occurs through mechanism-based inhibition, which are dose-dependent time-dependent and NADPH-dependent inhibition [21]. Probe cocktail assays can be employed to determine CYP450 enzyme kinetic parameters of inactivation (e.g., $\mathrm{k}_{\text {inact }}$, $\left.\mathrm{K}_{\mathrm{I}}, \mathrm{CL}_{\text {inact }}\right)[6]$.

\section{Conclusion}

Although there are challenges to be overcome, probe cocktail approach is a worthful solution for assessing in vitro DDIs of drug candidates. The development of analytical techniques such as UHPLC-MS/MS has allowed devising highly sensitive, selective and efficient assays, to avoid possible interactions among them and to analyze all the substrates and metabolites simultaneously. Probe cocktail approach in early drug development may be used in comprehensive DDI studies, providing faster and reliable results and improving the prediction and helping to prevent DDIs of the drug candidate.

\section{Financial \& competing interests disclosure}

The authors are grateful to the São Paulo Research Foundation (FAPESP, grant numbers: 2016/15680-5 and 2018/075344), Fundação de Amparo à Pesquisa do Estado do Amazonas (FAPEAM - Edital Universal Amazonas, Process number: 062.00343/2013), Conselho Nacional de Desenvolvimento Científico e Tecnológico (CNPq - INCT-DATREM Process number 465571/2014-0) and Coordenação de Aperfeiçoamento de Pessoal de Nível Superior (CAPES). The authors have no other relevant affiliations or financial involvement with any organization or entity with a financial interest in or financial conflict with the subject matter or materials discussed in the manuscript apart from those disclosed.

No writing assistance was utilized in the production of this manuscript.

\section{References}

1. US FDA. In vitro metabolism- and transporter-mediated drug-drug interaction studies - guidance for industry. 1-47 (2017). https://www.fda.gov/downloads/Drugs/Guidances/UCM581965.pdf

2. Lee B, Ji H, Lee T, Liu K. Simultaneous screening of activities of five cytochrome P450 and four uridine 5'-diphospho-glucuronosyltransferase enzymes in human liver microsomes using cocktail incubation and liquid chromatography-tandem mass spectrometry. Drug Metab. Dispos. 43, 1137-1146 (2015).

3. Spaggiari D, Daali Y, Rudaz S. An extensive cocktail approach for rapid risk assessment of in vitro CYP450 direct reversible inhibition by xenobiotic exposure. Toxicol. Appl. Pharmacol. 302, 41-51 (2016).

4. Chen Z, Zhang S, Long $\mathrm{N}$ et al. An improved substrate cocktail for assessing direct inhibition and time-dependent inhibition of multiple cytochrome P450s. Acta Pharmacol. Sin. 37(5), 708-718 (2016).

5. Ramanathan R, Ghosal A, Ramanathan L, Comstock K, Shen H, Ramanathan D. HPLC-high-resolution mass spectrometry with polarity switching for increasing throughput of human in vitro cocktail drug-drug interaction assay. Bioanalysis 10(9), 659-672 (2018).

6. Dahlinger D, Duechting S, Nuecken D, Sydow K, Fuhr U, Frechen S. Development and validation of an in vitro, seven-in-one human cytochrome P450 assay for evaluation of both direct and time-dependent inhibition. J. Pharmacol. Toxicol. Methods 77, 66-75 (2016).

7. Spaggiari D, Geiser L, Rudaz S. Coupling ultra-high-pressure liquid chromatography with mass spectrometry for in-vitro drug-metabolism studies. Trends Anal. Chem. 63, 129-139 (2014).

8. Grangeon A, Gravel S, Gaudette F, Turgeon J, Michaud V. Highly sensitive LC-MS/MS methods for the determination of seven human CYP450 activities using small oral doses of probe-drugs in human. J. Chromatogr. B 1040, 144-158 (2017).

9. Pillai VC, Strom SC, Caritis SN, Venkataramanan R. A sensitive and specific CYP cocktail assay for the simultaneous assessment of human cytochrome P450 activities in primary cultures of human hepatocytes using LC-MS/MS. J. Pharm. Biomed. Anal. 74, 126-132 (2013). 
10. Dierks EA, Stams KR, Lim H, Cornelius G, Zhang H, Ball SE. A method for the simultaneous evaluation of the activities of seven major human drug-metabolizing cytochrome P450s using an in vitro cocktail of probe substrates and fast graditent liquid chromatography tandem mass spectrometry. Drug Metab. Dispos. 29(1), 23-29 (2001).

11. US FDA. Drug development and drug interactions: Table of substrates, inhibitors, and inducers. 1-17 (2015). http://www.fda.gov/Drugs/DevelopmentApprovalProcess/DevelopmentResources/DrugInteractionsLabeling/ucm093664.htm

12. Peng $\mathrm{Y}$, Wu $\mathrm{H}$, Zhang $\mathrm{X}$ et al. A comprehensive assay for nine major cytochrome $\mathrm{P} 450$ enzymes activities with 16 probe reactions on human liver microsomes by a single LC/MS/MS run to support reliable in vitro inhibitory drug-drug interaction evaluation. Xenobiotica 45(11), 961-977 (2015).

13. Spaggiari D, Mehl F, Desfontaine V et al. Comparison of liquid chromatography and supercritical fluid chromatography coupled to compact single quadrupole mass spectrometer for targeted in vitro metabolism assay. J. Chromatogr. A 1371, 244-256 (2014).

14. Oh H, Lee H, Kim D, Hwa B. Development of GC-MS based cytochrome P450 assay for the investigation of multi-herb interaction. Anal. Biochem. 519, 71-83 (2017).

15. Qin C, Ren X, Tan Z, Chen Y, Yin J. A high-throughput inhibition screening of major human cytochrome P450 enzymes using an in vitro cocktail and liquid chromatography - tandem mass spectrometry. Biomed. Chromatogr. 28, 197-203 (2014).

16. González-Ruiz V, Olives AI, Martín MA. Core-shell particles lead the way to renewinghigh-performance liquid chromatography. Trends Anal. Chem. 64, 17-28 (2015).

17. O'Sullivan GP, Scully NM, Glennon JD. Polar-embedded and polar-endcapped stationary phases for LC. Anal. Lett. 43, 1609-1629 (2010).

18. Li G, Huang K, Nikolic D, Breemen RB Van. High-throughput cytochrome P450 cocktail inhibition assay for assessing drug-drug and drug-botanical interactions. Drug Metab. Dispos. 43, 1670-1678 (2015).

19. EMA (European Medicines Agency). Guideline on bioanalytical method validation. EMEA, Comm. Med. Prod. Hum. Use. 44, 1-23 (2012).

20. Bonfilio R, Cazedey ECL, de Araújo MB, Salgado HRN. Analytical validation of quantitative high-performance liquid chromatographic methods in pharmaceutical analysis: a practical approach. Crit. Rev. Anal. Chem. 42(1), 87-100 (2012).

21. Ogilvie BW, Usuki E, Phyllis Y, Parkinson A. In vitro approaches for studying the inhibition of drug-metabolizing enzymes and identifying the drug-metabolizing enzymes responsible for the metabolism of drugs (reaction phenotyping) with emphasis on cytochrome P450. In: Drug-Drug Interactions. Rodrigues AD (Ed.). CRC Press, FL, USA 213-358 (2008). 